\title{
Precise Control of $\pi$-electron Magnetism in Metal-free Porphyrins
}

\author{
Yan Zhao ${ }^{1 \dagger}$, Kaiyue Jiang ${ }^{2 \dagger}$, Can $\mathrm{Li}^{1}$, Yufeng Liu ${ }^{1}$, Chengyang Xu${ }^{1}$, Wenna Zheng ${ }^{1}$, Dandan Guan ${ }^{1,3,4}$, Yaoyi \\ $\mathrm{Li}^{1,3,4}$, Hao Zheng ${ }^{1,3,4}$, Canhua Liu ${ }^{1,3,4}$, Weidong Luo ${ }^{1,5}$, Jinfeng Jia ${ }^{1,3,4}$, Xiaodong Zhuang ${ }^{2 *}$, Shiyong Wang ${ }^{1,3,4 *}$ \\ ${ }^{1}$ Key Laboratory of Artificial Structures and Quantum Control (Ministry of Education), Shenyang National \\ Laboratory for Materials Science, School of Physics and Astronomy, Shanghai Jiao Tong University, Shanghai \\ 200240, China \\ ${ }^{2}$ The meso-Entropy Matter Lab, School of Chemistry and Chemical Engineering, Shanghai Jiao Tong University, \\ Shanghai 200240, China \\ ${ }^{3}$ Tsung-Dao Lee Institute, Shanghai Jiao Tong University, Shanghai, 200240, China. \\ ${ }^{4}$ Shanghai Research Center for Quantum Sciences, Shanghai 201315, China \\ ${ }^{5}$ Institute of Natural Sciences, Shanghai Jiao Tong University, Shanghai, 200240, China. \\ ${ }^{\dagger}$ These authors contributed equally to this work. \\ *Corresponding Authors: shiyong.wang@sjtu.edu.cn, zhuang@sjtu.edu.cn
}

\begin{abstract}
The porphyrin marcocycle can stabilize a set of magnetic metal ions, thus introducing localized net spins near the center. However, it remains illusive but most desirable to introduce delocalized spins in porphyrins with wide implications, for example, for building correlated quantum spins. Here, we demonstrate that metal-free porphyrins host delocalized $\pi$-electron magnetism as revealed by scanning probe microscopy and different level of theory calculations. Our results demonstrate that engineering of $\pi$-electron topologies introduces spin polarized singlet state and delocalized net spins in metal-free porphyrins. In addition, the $\pi$-electron magnetism can be switched on/off via STM manipulation by tunning the interfacial charge transfer. Our results provide an effective way to precisely control the $\pi$-electron magnetism in metal-free porphyrins, which can be further extended to design new magnetic functionalities of porphyrin-based architectures.
\end{abstract}




\section{INTRODUCTION}

Porphyrin molecules have a wide spectrum of physicochemical and biological properties with diversed applications in sensors, molecular electronics/spintronics and medical applications ${ }^{1-3}$. The rich functionalities of porphyrin complexes orginate from the center macrocycle, which can stablize a variety of elements inside and thus gives rise to distinct structural, electronic, magnetic, optoelectronic, and transport properties ${ }^{4-13}$. Magnetic metalloporphyrins have been extensively studied by various techniques, exhiting kondo and spin excitation effects, Yu-Shiba bound states, and spin-state switching ${ }^{13-27}$. Recently, hybridized graphene and metalloporphyrin nanostructures have been achieved, hosting combined intriguing electronic and magnetic properties ${ }^{28-33}$. The magnetism of metalloporphyrin architectures originates from the stabilized metal ions, and thus localizes at the center of the molecules. It remains elusive but most desirable to develop strategies to introduce delocalized spins in porphyrins for designer new magnetic functionalities in porphyrin architectures.

Recent advances of on-surface synthesis allow for tailoring $\pi$-electron topologies at the single chemical bond level, and thus provide the ability to precisely engineer $\pi$-electron magnetism in low-dimensional graphene nanostructures by introducing sublattice imbalance, topological frustration and topological defects ${ }^{34-50}$. Examples like triangulenes $s^{41,43,48,51}$, bowtie-shape nanographenes ${ }^{47}$, chiral graphene nanoribbons ${ }^{45}$, polymers ${ }^{52}$ and nanographenes with topological defects ${ }^{46,49}$ have been recently synthesized and characterized on surfaces. Similar as nanographenes, the porphyrin macrocycle is aromatic and can be interpreted as a multiple-bridged aromatic diaza[18]annulene system ${ }^{53,54}$. Thorough tailoring peripheral $\pi$-electron topology, it is in pricinple able to introduce tunable $\pi$-electron magnetism in metal-free porphyrin architectures, thus greatly enriching magnetic functionalities of porphyrin-based systems. Although substituted open-shell porphyrins have been realized using conventional solution synthesis methods, on-surface synthesis of unsubstituted porphyrins with $\pi$-electron magnetism remains to be explored ${ }^{55,56}$.

Here, we demonstrate such ability to precisely control delocalized magnetism in metal-free porphyrins through tailoring their peripheral $\pi$-electron topologies. Using combined solution and on-surface synthesis, three metalfree porphyrins with different $\pi$-electron topologies have been synthesized. Their chemical structures have been resolved by using high resolution non-contact atomic force microscopy, permitting us to clearly distinguish their $\pi$-electron topologies at the single chemical bond level. Using low-temperature scanning tunneling spectroscopy, their $\pi$-electron magnetism has been confirmed by Kondo effects. The experimental observations can be nicely elucidated by clar empirical rule, mean-field Hubbard model and spin-polarized density functional theory calculations. In addition, the $\pi$-electron magnetism can be reversibly switched on/off via STM manipulation by 
tunning the charge transfer from the underneath metal substrate. The realization of $\pi$-electron magnetism in metalfree porphyrin may be further extended to build correlated spins in porphyrin-based nanostructures, with implications for quantum information and spintronics applications.

\section{RESULTS AND DISCUSSION}

Using combined in-solution and on-surface synthesis, metal-free porphyrins with different $\pi$-electron topologies have been synthesized. As shown in Fig. 1a, the precursor 5,10,15,20-tetrakis(2,6-dimethylphenyl)porphyrin is synthesized in solution by using freshly distilled pyrrole and 2,6-dimethylbenzaldehyde (cf. detailed synthesis method in supplementary fig. 1). After in-solution synthesis, the precursor is thermally deposited on a Au(111) substrate held at room temperature following a subsequent annealling to $295{ }^{\circ} \mathrm{C}$ for 10 minutes. At elevated temperature, thermal-induced cyclodehydrogenation of methyl units occurs, giving rise to fully aromatic porphyrins ${ }^{39,57}$. Except for the main cyclodehydrogenation, two common side reactions take place occationally on $\mathrm{Au}(111)$ : i) few methyl units detach from the precursors forming five-membered carbon rings after subsequent cyclodehydrogenation, and ii) partial dehydrogenation sometimes occurs and introduces $S P_{3}$ hybridized carbon sites at edges. As shown in Fig. 1b, three products have been frequently observed and characterized. The Product \#1 contails two pentagon carbon-rings at opposite sides, and the Product \#3 has a $S P_{3}$ hybridized carbon site passivated by two hydrogen atoms. Their chemical structures have been clearly resolved by high resolution ncAFM imaging using a CO-functionalized tip (cf. Fig. 1c-e). The number of unpaired $\pi$-electrons (radicals) in the resulted porphyrins has been determined by using Clar's empirical rule (cf. all the possible $\pi$-electron networks in supplementary Fig. 3 and 4) $)^{58,59}$. As shown in Fig. 1, the Product \#1 is a close-shell molecule, while the Product \#2 and \#3 have two and one unpaired $\pi$-electron(s), repectively (as marked by red dots in Fig. 1). The presence of radicals suggests an open-shell configuration in the product $\# 2$ and $\# 3$, thus giving rise to delocalized $\pi$-electron magnetism.

The energy spectra and frontier molecular orbitals of the resulted porphyrins have been calculated by using meanfield Hubbard model (MFHM) and spin-polarzied density functional theory (SP-DFT), which have been widely used to determine the magnetic structure of nanographenes and show nice agreements with experiments ${ }^{37}$. As shown in Fig. 2a-c, the Product \#1 has a close-shell ground state as confirmed by combined Clar empirical rule, MFHM and SP-DFT calculations. We notice that MFHM calculations qualitatively agree with SP-DFT, despite the exact energy position of each state varies a little bit from each other. Experimentally, dfferential conductance $(\mathrm{dI} / \mathrm{dV})$ spectroscopy has been used to detect the electronc structure of achieved porphyrins. Together with spatial mappings, $\mathrm{dI} / \mathrm{dV}$ spectroscopy is able to resolve both the energy positions and spatial distribution of molecular 
orbitals with ultra-high energy and spatial resolution. As shown in Fig. 2d, dI/dV spectra taken above the center of the Product \#1 reveal two resonances at $-1.4 \mathrm{~V}$ and $0.9 \mathrm{~V}$, which can be assigned to the highest occupied molecular orbital (HOMO) and lowest unoccupied molecular orbital (LUMO), respectively. dI/dV mappings at these two energyies resolve the spatial distribution of these two molecular states, which agree well with HOMO and LUMO orbital shapes as calculated by MFHM and SP-DFT. These results confirm that the Product \#1 is a close-shell molecule without any unpaired $\pi$-electrons.

Despite its slightly different $\pi$-electron topology from that of the Product $\# 1$, the Product \#2 has drastically different magnetic ground state. The Product \#2 has in total $58 \pi$-elelctrons, with $2 \pi$-electrons more than those of the product \#1. Although both of them host even number of $\pi$-electrons, the Product \#2 has an open-shell electronic structure as opposed to the close-shell configuration of the Product \#1 (cf. Fig. 3a). The reason is due to topological frustration, that is, it is impossible to concomitantly pair all $\mathrm{p}_{z}$ orbitals to form $\pi$ bonds in the Product \#2. This picture can be verified by both MFHM and SP-DFT calculations as shown in figure 3b-e. The calculated energy levels in Fig. 3b-c show that there are two degenerated states below fermi level, which are spatially separated with enhanced orbital density at opposite sides (cf. Fig. 3d and e), and singly occupied by two electrons with oppositely oriented spins. In other words, the ground electronic configuration breaks spin-spatial symmetry and exhibits antiferromagnetic ordering, which can be nicely seen in the calculated spin density maps in Fig. $3 \mathrm{f}$ and g. The mechanism behind is very similar to Clar Goblet, where topological frustration induces spin polarized singlet state in the bowtie-shape nanographene $e^{36,47}$.

However, experimental $\mathrm{dI} / \mathrm{dV}$ measurements reveal that the Product \#2 hosts a net spin of $\mathrm{S}=1 / 2$ by showing a sharp Kondo resonances instead of the expected singlet ground state with a net spin of $\mathrm{S}=0$ (cf. Fig. 4g). As illustrated in Fig. 4a, we attribute this discrepancy to the interfacial charge transfer, where one electron is transferred from molecule to gold substrate making it positively charged. Similar charging effects have been previously observed in several graphene nanostructures, such as the zigzag termina of $\mathrm{N}=7$ armchair graphene nanoribbons ${ }^{60,61}$, and $\mathrm{N}=6$ zigzag graphene nanoribbon ${ }^{39}$. As shown in Fig. $4 \mathrm{~b}-\mathrm{c}$, the electronic structure of the positively charged Product \#2 is calculated by MFHM and SP-DFT, revealing a singly occupied molecular state below fermi level. This means the charged molecule is with a net magnetic moment of $S=1 / 2$. On metal substrate, this magnetic moment will be screened by $\mathrm{Au}(111)$ electron reservoir, which can be understood by single impurity Anderson Model ${ }^{17,62}$. As shown in Fig. 4g, dI/dV spectra reveal such screening effect by showing a sharp Kondo resonance at Fermi level. In addition, the observed spin density distribution nicely agrees with calculated results, further supporting that the observed zero-bias peak originates from Kondo resonance (cf. Fig. 4e-f). As per 
Anderson model, the Kondo peak width varies with temperature with full width at half maximum (FWHM) of $\Gamma=\sqrt{\left(\alpha k_{B} T\right)^{2}+\left(2 k_{B} T_{K}\right)^{2}}$, where $\mathrm{T}$ is the temperature, $T_{K}$ is the Kondo temperature, and $\alpha$ is the slope of linear growth of the width at $T \gg T_{K}$. The experimental obtained temperature dependent FWHM can be well fitted by the previous equation, giving a Kondo temperature of $22 \mathrm{~K}$ (cf. Fig 4h-i).

Using similar techniques, the magnetic properties of the Product \#3 has been determined. Since one carbon site is passivated by two hydrogen atoms, the Product \#3 has one $\pi$-electron less than the Product \#2 with an odd number of $57 \pi$-electrons in total. It is impossible to concomitantly pair odd number of $\mathrm{p}_{z}$ orbitals to form $\pi$ bonds, thus generating one $\pi$ radical as marked by a red dot in Fig. 5a. MFHM and SP-DFT calculations confirm the presence of a singly occupied state below fermi level in the Prodcut \#2, that is, a net spin of $S=1 / 2$. As shown in Fig. $5 d$, the passivation of $\pi$-radical locally modifies its spin density distribution, which gets significantly quenched near the $\mathrm{CH}_{2}$ site (cf. Clar non-kekulé structures in Supplementary Fig. 3). The emergence of net spins in porphyrins with odd number of $\pi$ electrons is very similar to nanographenes with sublattice imbalance as proposed by E. Lieb in $1989^{34}$. As shown in Fig. 5g, dI/dV spectroscopy measurements confirm the emergence of a net spin of $\mathrm{S}=1 / 2$ in the product \#3. The net spin couples with underneath electron resorviour, genenrating Kondo resonances by showing a sharp Fano-shape peak at fermi level in dI/dV spectra with peak intensity proportional to spin density magnitude. In addition, the resovled spin density distribution fits very well with the calculated result (cf. Fig. 5e and f). The nice agreements between experiments and calculations in gas phase indicate that the Product \#3 is neutral on $\mathrm{Au}(111)$.

Although the Product \#3 shares very similar adsorption geometries with that of the Product \#2, the former is neutral on $\mathrm{Au}(111)$ while the later is positively charged. By carefully checking the AFM images in Fig. 1c-e, one can see that the presence of $S P_{3}$ carbon $\left(\mathrm{CH}_{2}\right)$ site slightly lifts the coner near the $\mathrm{CH}_{2}$ site away from the surface, which is imaged as a local bright protrusion in nc-AFM imaging due to a stronger Pauli repulsion. This slight adsorption difference leads to a completely different charging behavior. Previous DFT calculations by using a set of van der Waals correction schemes reveal that despite very close adsorption heights of most graphene nanostructures on $\mathrm{Au}(111)$, the magnetism (charge transfer) is very sensitive to small perturbations (adsorption height variations), supporting our observations ${ }^{63}$. To gain more evidences of the charging effect, we performed STM manipulation to locally modify the adsorption configurations. After each manipulation, we performed nc-AFM imaging, constantheight current imaging and $\mathrm{dI} / \mathrm{dV}$ spectroscopy to trace the charging transfer. As shown in fig. 6a, the porphyrin molecule is initially neutral by showing a Kondo resonance at Fermi level. After manipulation, the molecule rotates an angle of $185^{\circ}$ around the out-of-plane axis (cf. Fig. 6b). Although nc-AFM and constant-height current image 
indicate a similar adsorption geometry after rotation, $\mathrm{dI} / \mathrm{dV}$ spectrascopy reveals that the molecule is positively charged by showing a broad peak above fermi level instead of exhibiting a sharp Kondo resonance at fermi level (cf. the No. 2 spectrum in Fig. 6d). In other words, the electron previously occupied the singly occupied state transfers to gold substrate, thus making the molecule positively charged and leading all oribitals doubly occupied. Further manipulation experiment was used to rotate the molecule again. As shown in Fig. 6c, the Kondo resonance appears after a successive rotation of $125^{\circ}$, suggesting the molecule becomes neutral again. In total, we performed successive 9 times STM manipulations, and found that the charging effect is extremely sensitively to adsorption variations (cf. supplementary Fig.8). Similar manipulation experiments have been performed on the Product \#1 and \#2 without obtaining this switching effect. These results indicate that slightly decoupling the molecule from surface can effectively quench the charging effect, such as by lifting the molecule slight away from the substrate by functional groups.

\section{CONCLUSION}

In conclusion, we demonstrate an effective approach to engineer $\pi$-electron magnetism in metal-free porphyrins by using combined non-contact atomic force microscopy, scanning tunneling microscopy/spectroscopy as well as theory calculations at different levels. Three types of porphyrins with different $\pi$-electron topologies have been successively synthesized and characterized down to the single chemical bond level. The $\pi$-electron network in porphyrins with even number of $\pi$ electrons can either host a close-shell configuration or spin polarized singlet ground state depending whether the network gets frustrated or not. Porphyrins with odd number of $\pi$ electrons host nets spin similar as sublattice imbalance induced spins in nanographenes. In addition, the magnetism can be switched on/off by tuning charge transfer from underneath $\mathrm{Au}(111)$. Our method reported herein provides ample opportunities for further designer correlated spins in porphyrin-based nanostructures to study their many-body effects. 


\section{ASSOCIATED CONTENT}

Supporting Information. Detailed descriptions of experimental and theoretical procedures, Clar non-kekulé structures of the product \#2 and 3, Magnetic filed dependence of Kondo resonance, dI/dV spectra and mappings, and simulated local density of state maps. This material is available free of charge via the Internet at http://pubs.acs.org.

\section{AUTHOR INFORMATION}

Yan Zhao and Kaiyue Jiang contributed equally to this work.

\section{ACKNOWLEDGEMENT}

S. W. acknowledges the financial support from the National Natural Science Foundation of China (No. 11874258), the Shanghai Municipal Science and Technology Qi Ming Xing Project (No. 20QA1405100) and Fok Ying Tung Foundation for young researchers. This work is also supported by the Ministry of Science and Technology of China (Grants No. 2016YFA0301003, No. 2016YFA0300403), the National Natural Science Foundation of China (Grants No. 11521404, No. 11634009, No. 11574202, No. 11874256, No. 11790313, No. 11674226, No. U1632102, No. 11674222, No. U1632272 and No. 11861161003), the Strategic Priority Research Program of Chinese Academy of Sciences (Grant No. XDB28000000), and the Shanghai Municipal Science and Technology Major Project (Grant No.2019SHZDZX01). Y.L. thanks ECNU Multifunctional Platform for Innovation (001) of East China Normal University for providing computing resources. 


\section{REFERENCES}

(1) Kadish, K.; Smith, K.; Guilard, R. Handbook of Porphyrin Science; World Scientific Publishing Company, 2010.

(2) Senge, M. O.; Fazekas, M.; Notaras, E. G. A.; Blau, W. J.; Zawadzka, M.; Locos, O. B.; Ni Mhuircheartaigh, E. M. Nonlinear Optical Properties of Porphyrins. Adv. Mater. 2007, 19 (19), 27372774.

(3) Huang, H.; Song, W.; Rieffel, J.; Lovell, J. F. Emerging Applications of Porphyrins in Photomedicine. Front. Phys. 2015, 3, 23.

(4) Baklanov, A.; Garnica, M.; Robert, A.; Bocquet, M.-L.; Seufert, K.; Küchle, J. T.; Ryan, P. T. P.; Haag, F.; Kakavandi, R.; Allegretti, F.; Auwärter, W. On-Surface Synthesis of Nonmetal Porphyrins. J. Am. Chem. Soc. 2020, 142 (4), 1871-1881.

(5) Auwärter, W.; Écija, D.; Klappenberger, F.; Barth, J. V. Porphyrins at Interfaces. Nat. Chem. 2015, 7 (2), $105-120$.

(6) Grill, L.; Dyer, M.; Lafferentz, L.; Persson, M.; Peters, M. V.; Hecht, S. Nano-Architectures by Covalent Assembly of Molecular Building Blocks. Nat. Nanotechnol. 2007, 2 (11), 687-691.

(7) Gottfried, J. M.; Flechtner, K.; Kretschmann, A.; Lukasczyk, T.; Steinrück, H.-P. Direct Synthesis of a Metalloporphyrin Complex on a Surface. J. Am. Chem. Soc. 2006, 128 (17), 5644-5645.

(8) Zhang, Y.; Luo, Y.; Zhang, Y.; Yu, Y.-J.; Kuang, Y.-M.; Zhang, L.; Meng, Q.-S.; Luo, Y.; Yang, J.-L.; Dong, Z.-C.; Hou, J. G. Visualizing Coherent Intermolecular Dipole-Dipole Coupling in Real Space. Nature 2016, 531 (7596), 623-627.

(9) Kumagai, T.; Hanke, F.; Gawinkowski, S.; Sharp, J.; Kotsis, K.; Waluk, J.; Persson, M.; Grill, L. Controlling Intramolecular Hydrogen Transfer in a Porphycene Molecule with Single Atoms or Molecules Located Nearby. Nat. Chem. 2014, 6 (1), 41-46.

(10) Zhao, A.; Li, Q.; Chen, L.; Xiang, H.; Wang, W.; Pan, S.; Wang, B.; Xiao, X.; Yang, J.; Hou, J. G.; Zhu, Q. Controlling the Kondo Effect of an Adsorbed Magnetic Ion Through Its Chemical Bonding. Science 2005, 309 (5740), 1542-1544.

(11) Chen, X.; Fu, Y.-S.; Ji, S.-H.; Zhang, T.; Cheng, P.; Ma, X.-C.; Zou, X.-L.; Duan, W.-H.; Jia, J.-F.; Xue, Q.-K. Probing Superexchange Interaction in Molecular Magnets by Spin-Flip Spectroscopy and Microscopy. Phys. Rev. Lett. 2008, 101 (19), 197208.

(12) Kuang, G.; Chen, S.-Z.; Wang, W.; Lin, T.; Chen, K.; Shang, X.; Liu, P. N.; Lin, N. Resonant Charge Transport in Conjugated Molecular Wires beyond $10 \mathrm{Nm}$ Range. J. Am. Chem. Soc. 2016, 138 (35), 11140-11143.

(13) Köbke, A.; Gutzeit, F.; Röhricht, F.; Schlimm, A.; Grunwald, J.; Tuczek, F.; Studniarek, M.; Longo, D.; Choueikani, F.; Otero, E.; Ohresser, P.; Rohlf, S.; Johannsen, S.; Diekmann, F.; Rossnagel, K.; Weismann, A.; Jasper-Toennies, T.; Näther, C.; Herges, R.; Berndt, R.; Gruber, M. Reversible Coordination-Induced Spin-State Switching in Complexes on Metal Surfaces. Nat. Nanotechnol. 2020, 15 (1), 18-21.

(14) Girovsky, J.; Nowakowski, J.; Ali, Md. E.; Baljozovic, M.; Rossmann, H. R.; Nijs, T.; Aeby, E. A.; Nowakowska, S.; Siewert, D.; Srivastava, G.; Wäckerlin, C.; Dreiser, J.; Decurtins, S.; Liu, S.-X.; Oppeneer, P. M.; Jung, T. A.; Ballav, N. Long-Range Ferrimagnetic Order in a Two-Dimensional Supramolecular Kondo Lattice. Nat. Commun. 2017, 8 (1), 15388.

(15) Wang, W.; Pang, R.; Kuang, G.; Shi, X.; Shang, X.; Liu, P. N.; Lin, N. Intramolecularly Resolved Kondo Resonance of High-Spin Fe-Porphyrin Adsorbed on Au(111). Phys. Rev. B 2015, 91 (4), 045440.

(16) Kezilebieke, S.; Žitko, R.; Dvorak, M.; Ojanen, T.; Liljeroth, P. Observation of Coexistence of Yu-ShibaRusinov States and Spin-Flip Excitations. Nano Lett. 2019, 19 (7), 4614-4619.

(17) Ternes, M.; Heinrich, A. J.; Schneider, W.-D. Spectroscopic Manifestations of the Kondo Effect on Single Adatoms. J. Phys. Condens. Matter 2008, 21 (5), 053001.

(18) Ternes, M. Spin Excitations and Correlations in Scanning Tunneling Spectroscopy. New J. Phys. 2015, 17 (6), 063016.

(19) Choi, D.-J.; Lorente, N.; Wiebe, J.; von Bergmann, K.; Otte, A. F.; Heinrich, A. J. Colloquium: Atomic Spin Chains on Surfaces. Rev. Mod. Phys. 2019, 91 (4), 041001.

(20) Li, X.; Zhu, L.; Li, B.; Li, J.; Gao, P.; Yang, L.; Zhao, A.; Luo, Y.; Hou, J.; Zheng, X.; Wang, B.; Yang, J. Molecular Molds for Regularizing Kondo States at Atom/Metal Interfaces. Nat. Commun. 2020, 11 (1), 2566. 
(21) Liu, L.; Yang, K.; Jiang, Y.; Song, B.; Xiao, W.; Li, L.; Zhou, H.; Wang, Y.; Du, S.; Ouyang, M.; Hofer, W. A.; Castro Neto, A. H.; Gao, H.-J. Reversible Single Spin Control of Individual Magnetic Molecule by Hydrogen Atom Adsorption. Sci. Rep. 2013, 3 (1), 1210.

(22) Zhang, Q.; Kuang, G.; Pang, R.; Shi, X.; Lin, N. Switching Molecular Kondo Effect via Supramolecular Interaction. ACS Nano 2015, 9 (12), 12521-12528.

(23) Iancu, V.; Deshpande, A.; Hla, S.-W. Manipulating Kondo Temperature via Single Molecule Switching. Nano Lett. 2006, 6 (4), 820-823.

(24) Flechtner, K.; Kretschmann, A.; Steinrück, H.-P.; Gottfried, J. M. NO-Induced Reversible Switching of the Electronic Interaction between a Porphyrin-Coordinated Cobalt Ion and a Silver Surface. J. Am. Chem. Soc. 2007, 129 (40), 12110-12111.

(25) Tsukahara, N.; Shiraki, S.; Itou, S.; Ohta, N.; Takagi, N.; Kawai, M. Evolution of Kondo Resonance from a Single Impurity Molecule to the Two-Dimensional Lattice. Phys. Rev. Lett. 2011, 106 (18), 187201.

(26) Wäckerlin, C.; Chylarecka, D.; Kleibert, A.; Müller, K.; Iacovita, C.; Nolting, F.; Jung, T. A.; Ballav, N. Controlling Spins in Adsorbed Molecules by a Chemical Switch. Nat. Commun. 2010, 1 (1), 61.

(27) Li, R.; Li, N.; Wang, H.; Weismann, A.; Zhang, Y.; Hou, S.; Wu, K.; Wang, Y. Tuning the Spin-Related Transport Properties of FePc on Au(111) through Single-Molecule Chemistry. Chem. Commun. 2018, 54 (66), 9135-9138.

(28) Li, J.; Friedrich, N.; Merino, N.; de Oteyza, D. G.; Peña, D.; Jacob, D.; Pascual, J. I. Electrically Addressing the Spin of a Magnetic Porphyrin through Covalently Connected Graphene Electrodes. Nano Lett. 2019, $19(5), 3288-3294$.

(29) Li, J.; Merino-Díez, N.; Carbonell-Sanromà, E.; Vilas-Varela, M.; de Oteyza, D. G.; Peña, D.; Corso, M.; Pascual, J. I. Survival of Spin State in Magnetic Porphyrins Contacted by Graphene Nanoribbons. Sci. Adv. 2018, 4 (2) eaaq0582.

(30) Su, X.; Xue, Z.; Li, G.; Yu, P. Edge State Engineering of Graphene Nanoribbons. Nano Lett. 2018, 18 (9), 5744-5751.

(31) Mateo, L. M.; Sun, Q.; Liu, S.-X.; Bergkamp, J. J.; Eimre, K.; Pignedoli, C. A.; Ruffieux, P.; Decurtins, S.; Bottari, G.; Fasel, R.; Torres, T. On-Surface Synthesis and Characterization of Triply Fused PorphyrinGraphene Nanoribbon Hybrids. Angew. Chem. Int. Ed. 2020, 59 (3), 1334-1339.

(32) He, Y.; Garnica, M.; Bischoff, F.; Ducke, J.; Bocquet, M.-L.; Batzill, M.; Auwärter, W.; Barth, J. V. Fusing Tetrapyrroles to Graphene Edges by Surface-Assisted Covalent Coupling. Nat. Chem. 2017, 9 (1), 33-38.

(33) Bischoff, F.; He, Y.; Riss, A.; Seufert, K.; Auwärter, W.; Barth, J. V. Exploration of Interfacial Porphine Coupling Schemes and Hybrid Systems by Bond-Resolved Scanning Probe Microscopy. Angew. Chem. Int. Ed. 2018, 57 (49), 16030-16035.

(34) Lieb, E. H. Two Theorems on the Hubbard Model. Phys. Rev. Lett. 1989, 62 (10), 1201-1204.

(35) Yazyev, O. V.; Helm, L. Defect-Induced Magnetism in Graphene. Phys. Rev. B 2007, 75 (12), 125408.

(36) Wang, W. L.; Yazyev, O. V.; Meng, S.; Kaxiras, E. Topological Frustration in Graphene Nanoflakes: Magnetic Order and Spin Logic Devices. Phys. Rev. Lett. 2009, 102 (15), 157201.

(37) Yazyev, O. V. Emergence of Magnetism in Graphene Materials and Nanostructures. Rep. Prog. Phys. 2010, 73 (5), 056501.

(38) Ortiz, R.; Lado, J. L.; Melle-Franco, M.; Fernández-Rossier, J. Engineering Spin Exchange in Nonbipartite Graphene Zigzag Edges. Phys. Rev. B 2016, 94 (9), 094414.

(39) Ruffieux, P.; Wang, S.; Yang, B.; Sánchez-Sánchez, C.; Liu, J.; Dienel, T.; Talirz, L.; Shinde, P.; Pignedoli, C. A.; Passerone, D.; Dumslaff, T.; Feng, X.; Müllen, K.; Fasel, R. On-Surface Synthesis of Graphene Nanoribbons with Zigzag Edge Topology. Nature 2016, 531, 489.

(40) Gonzalez-Herrero, H.; Gomez-Rodriguez, J. M.; Mallet, P.; Moaied, M.; Palacios, J. J.; Salgado, C.; Ugeda, M. M.; Veuillen, J.-Y.; Yndurain, F.; Brihuega, I. Atomic-Scale Control of Graphene Magnetism by Using Hydrogen Atoms. Science 2016, 352 (6284), 437-441.

(41) Pavliček, N.; Mistry, A.; Majzik, Z.; Moll, N.; Meyer, G.; Fox, D. J.; Gross, L. Synthesis and Characterization of Triangulene. Nat. Nanotechnol. 2017, 31.

(42) Mishra, S.; Lohr, T. G.; Pignedoli, C. A.; Liu, J.; Berger, R.; Urgel, J. I.; Müllen, K.; Feng, X.; Ruffieux, P.; Fasel, R. Tailoring Bond Topologies in Open-Shell Graphene Nanostructures. ACS Nano 2018, 12 (12), 11917-11927.

(43) Mishra, S.; Beyer, D.; Eimre, K.; Liu, J.; Berger, R.; Gröning, O.; Pignedoli, C. A.; Müllen, K.; Fasel, R.; Feng, X.; Ruffieux, P. Synthesis and Characterization of $\pi$-Extended Triangulene. J. Am. Chem. Soc. 2019, 141 (27), 10621-10625. 
(44) Ortiz, R.; Boto, R. A.; García-Martínez, N.; Sancho-García, J. C.; Melle-Franco, M.; Fernández-Rossier, J. Exchange Rules for Diradical $\pi$-Conjugated Hydrocarbons. Nano Lett. 2019, 19 (9), 5991-5997.

(45) Li, J.; Sanz, S.; Corso, M.; Choi, D. J.; Peña, D.; Frederiksen, T.; Pascual, J. I. Single Spin Localization and Manipulation in Graphene Open-Shell Nanostructures. Nat. Commun. 2019, 10 (1), 200.

(46) Mishra, S.; Beyer, D.; Berger, R.; Liu, J.; Gröning, O.; Urgel, J. I.; Müllen, K.; Ruffieux, P.; Feng, X.; Fasel, R. Topological Defect-Induced Magnetism in a Nanographene. J. Am. Chem. Soc. 2020, 142 (3), 1147-1152.

(47) Mishra, S.; Beyer, D.; Eimre, K.; Kezilebieke, S.; Berger, R.; Gröning, O.; Pignedoli, C. A.; Müllen, K.; Liljeroth, P.; Ruffieux, P.; Feng, X.; Fasel, R. Topological Frustration Induces Unconventional Magnetism in a Nanographene. Nat. Nanotechnol. 2020, 15 (1), 22-28.

(48) Mishra, S.; Beyer, D.; Eimre, K.; Ortiz, R.; Fernández-Rossier, J.; Ber, R. Collective All-Carbon Magnetism in Triangulene Dimers. Angew. Chem. Int. Ed. 202059 (29), 12041-12047.

(49) Zheng, Y.; Li, C.; Zhao, Y.; Beyer, D.; Wang, G.; Xu, C.; Yue, X.; Chen, Y.; Guan, D.-D.; Li, Y.-Y.; Zheng, H.; Liu, C.; Luo, W.; Feng, X.; Wang, S.; Jia, J. Engineering of Magnetic Coupling in Nanographene. Phys. Rev. Lett. 2020, 124 (14), 147206.

(50) Yuan, B.; Li, C.; Zhao, Y.; Gröning, O.; Zhou, X.; Zhang, P.; Guan, D.; Li, Y.; Zheng, H.; Liu, C.; Mai, Y.; Liu, P.; Ji, W.; Jia, J.; Wang, S. Resolving Quinoid Structure in Poly(Para-Phenylene) Chains. J. Am. Chem. Soc. 2020, 142 (22), 10034-10041.

(51) Su, J.; Telychko, M.; Hu, P.; Macam, G.; Mutombo, P.; Zhang, H.; Bao, Y.; Cheng, F.; Huang, Z.-Q.; Qiu, Z.; Tan, S. J. R.; Lin, H.; Jelínek, P.; Chuang, F.-C.; Wu, J.; Lu, J. Atomically Precise Bottom-up Synthesis of $\pi$-Extended [5]Triangulene. Sci. Adv. 2019, 5 (7), 7717.

(52) Sánchez-Grande, A.; Urgel, J. I.; Cahlík, A.; Santos, J.; Edalatmanesh, S.; Rodríguez-Sánchez, E.; Lauwaet, K.; Mutombo, P.; Nachtigallová, D.; Nieman, R.; Lischka, H.; de la Torre, B.; Miranda, R.; Gröning, O.; Martín, N.; Jelínek, P.; Écija, D. Diradical Organic One-dimensional Polymers Synthesized on a Metallic Surface. Angew. Chem. Int. Ed. 2020, ange.202006276.

(53) Lash, T. D. Origin of Aromatic Character in Porphyrinoid Systems. J. Porphyr. Phthalocyanines 2011, 15 (11n12), 1093-1115..

(54) Zugermeier, M.; Herritsch, J.; Luy, J.-N.; Chen, M.; Klein, B. P.; Niefind, F.; Schweyen, P.; Bröring, M.; Schmid, M.; Tonner, R.; Gottfried, J. M. On-Surface Formation of a Transient Corrole Radical and Aromaticity-Driven Interfacial Electron Transfer. J. Phys. Chem. C 2020, 124 (25), 13825-13836.

(55) Zeng, W.; Lee, S.; Son, M.; Ishida, M.; Furukawa, K.; Hu, P.; Sun, Z.; Kim, D.; Wu, J. Phenalenyl-Fused Porphyrins with Different Ground States. Chem. Sci. 2015, 6 (4), 2427-2433.

(56) Shimizu, D.; Osuka, A. Porphyrinoids as a Platform of Stable Radicals. Chem. Sci. 2018, 9 (6), $1408-1423$.

(57) Beyer, D.; Wang, S.; Pignedoli, C. A.; Melidonie, J.; Yuan, B.; Li, C.; Wilhelm, J.; Ruffieux, P.; Berger, R.; Müllen, K.; Fasel, R.; Feng, X. Graphene Nanoribbons Derived from Zigzag Edge-Encased Poly(Para2,9-Dibenzo[Bc,Kl]Coronenylene) Polymer Chains. J. Am. Chem. Soc. 2019, 141 (7), 2843-2846.

(58) Sun, Z.; Wu, J. Open-Shell Polycyclic Aromatic Hydrocarbons. J. Mater. Chem. 2012, 22 (10), 41514160 .

(59) Clar, E. Polycyclic Hydrocarbons; Springer-Verlag Berlin Heidelberg, 1964

(60) van der Lit, J.; Boneschanscher, M. P.; Vanmaekelbergh, D.; Ijäs, M.; Uppstu, A.; Ervasti, M.; Harju, A.; Liljeroth, P.; Swart, I. Suppression of Electron-Vibron Coupling in Graphene Nanoribbons Contacted via a Single Atom. Nat. Commun. 2013, 4 (1), 2023.

(61) Wang, S.; Talirz, L.; Pignedoli, C. A.; Feng, X.; Müllen, K.; Fasel, R.; Ruffieux, P. Giant Edge State Splitting at Atomically Precise Graphene Zigzag Edges. Nat. Commun. 2016, 7, 11507.

(62) Kondo, J. Resistance Minimum in Dilute Magnetic Alloys. Prog. Theor. Phys. 1964, 32 (1), 37-49.

(63) Shinde, P. P.; Gröning, O.; Wang, S.; Ruffieux, P.; Pignedoli, C. A.; Fasel, R.; Passerone, D. Stability of Edge Magnetism in Functionalized Zigzag Graphene Nanoribbons. Carbon 2017, 124, 123-132. 
a

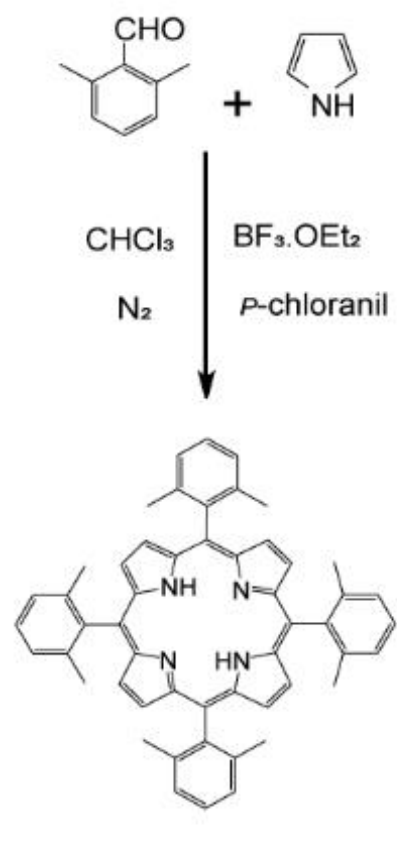

b
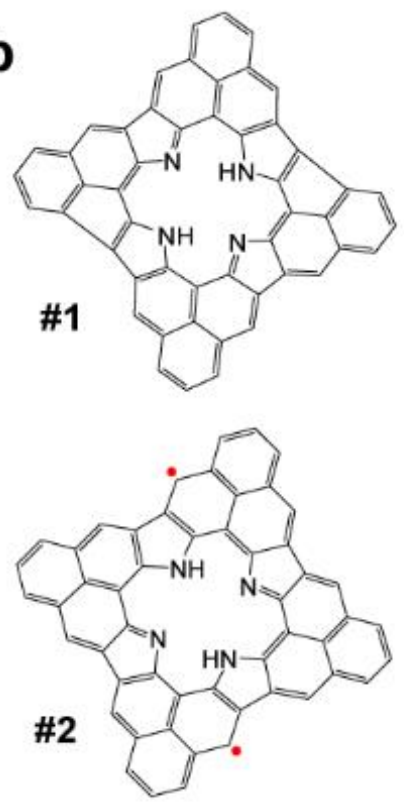

$\underset{\mathrm{Au}(111)}{\stackrel{295^{\circ} \mathrm{C}}{\longrightarrow}}$
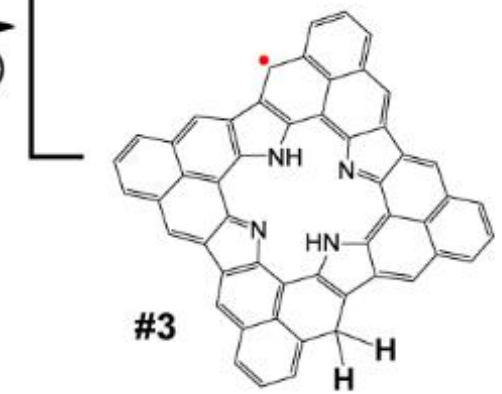
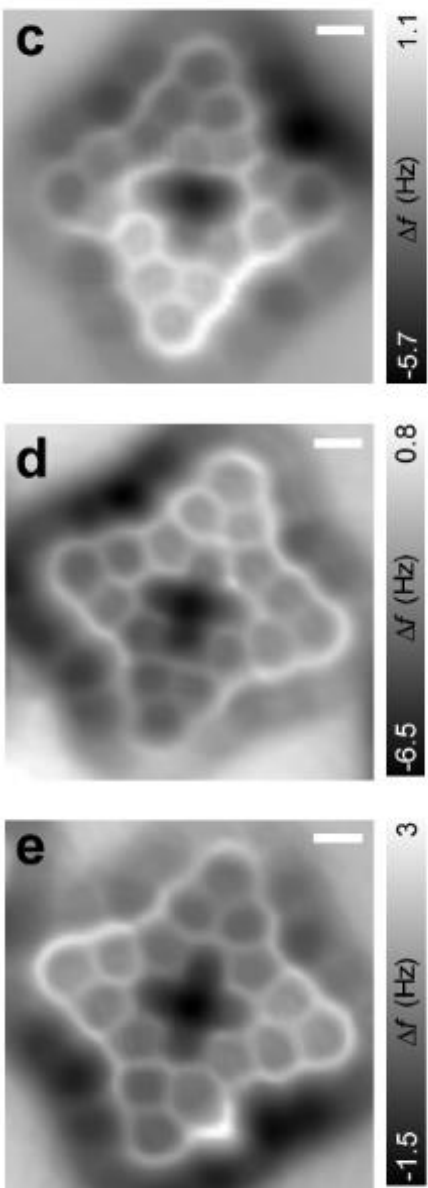

Figure 1. Synthesis of porphyrins with different $\pi$-electron topologies. (a), In-solution synthesis of molecular precursor 5,10,15,20-tetrakis(2,6-dimethylphenyl)porphyrin. (b), Chemical structures of three On-surface synthesized porphyrins with red dots indicating the unpaired $\pi$-electrons. (c-e), Nc-AFM frequency shift images (Resonant frequency: $26 \mathrm{KHz}$, Oscillation amplitude: $80 \mathrm{pm}$, Scale bars: $0.4 \mathrm{~nm}$ ) of the porphyrins in (b). 
a
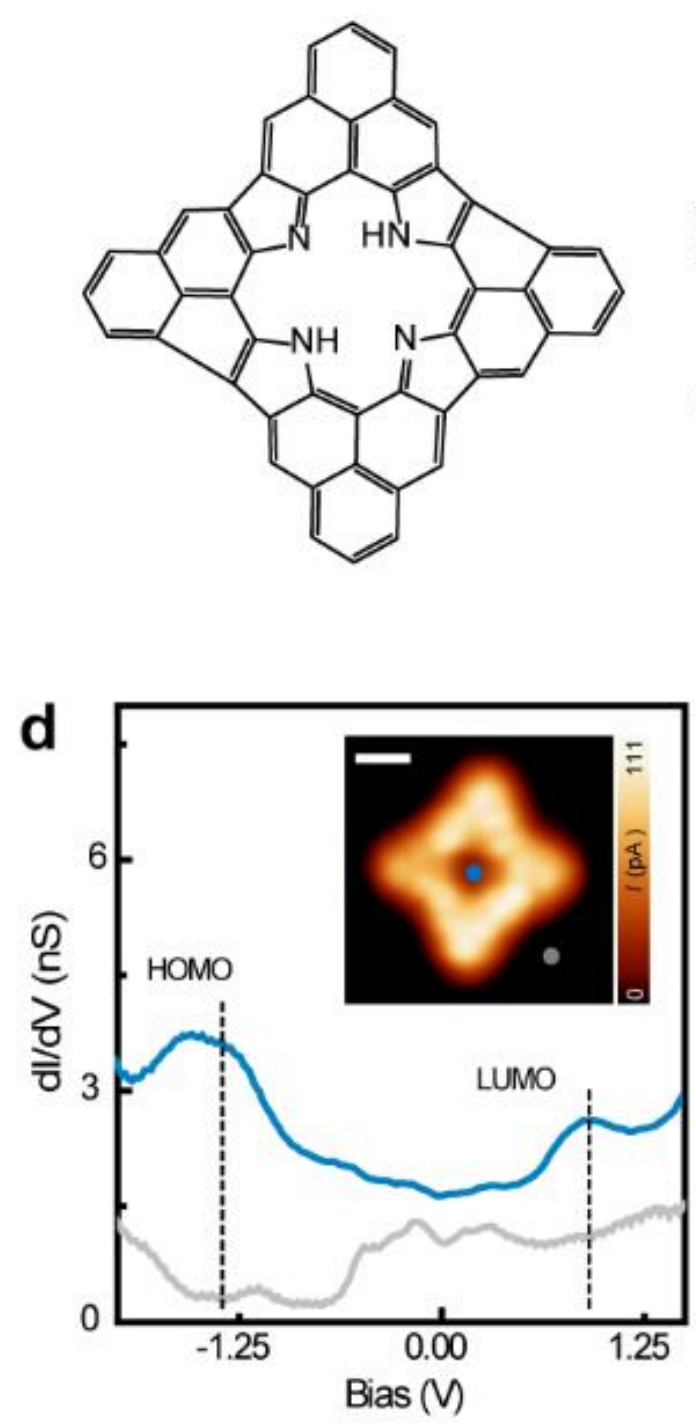
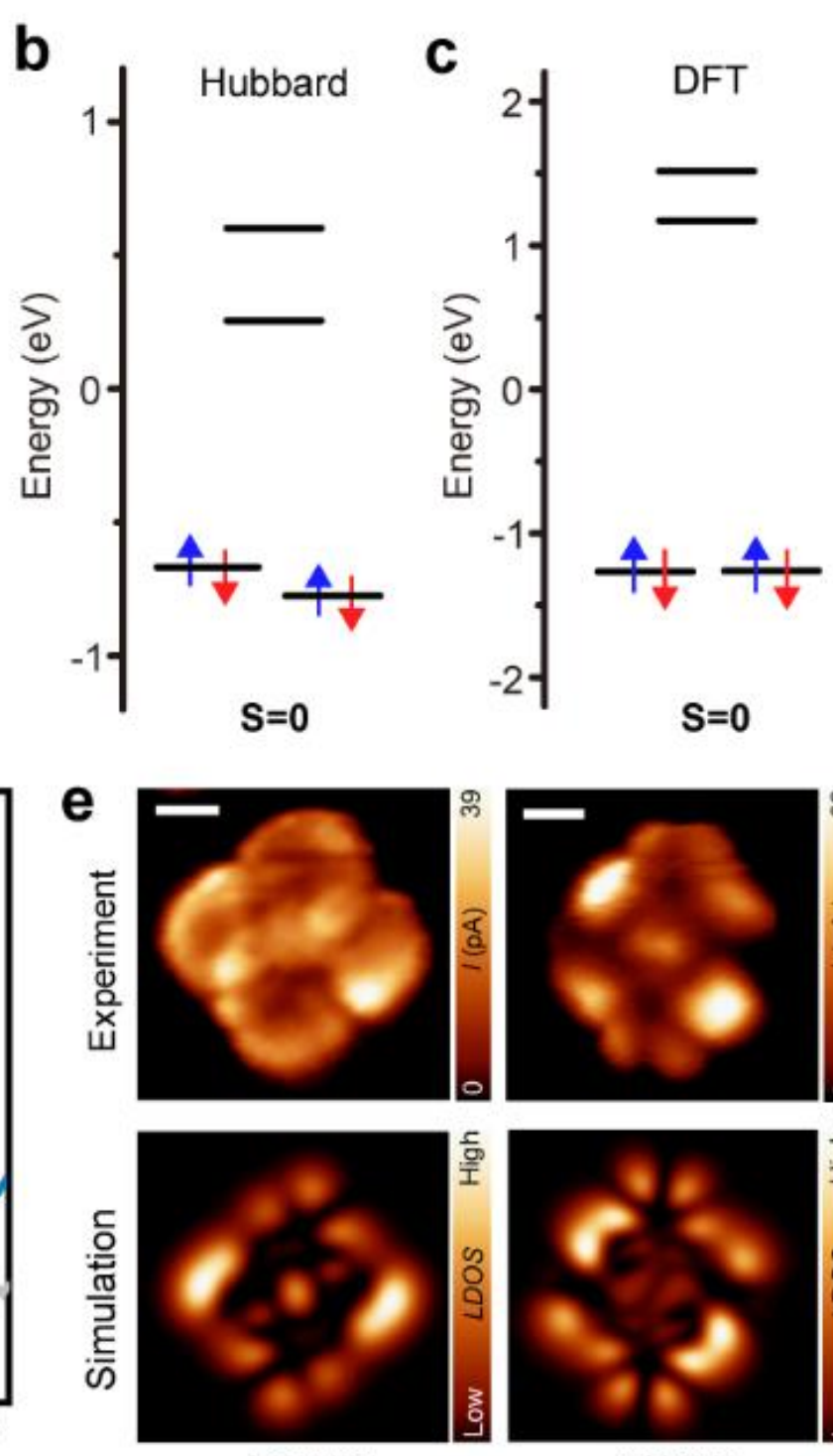

HOMO
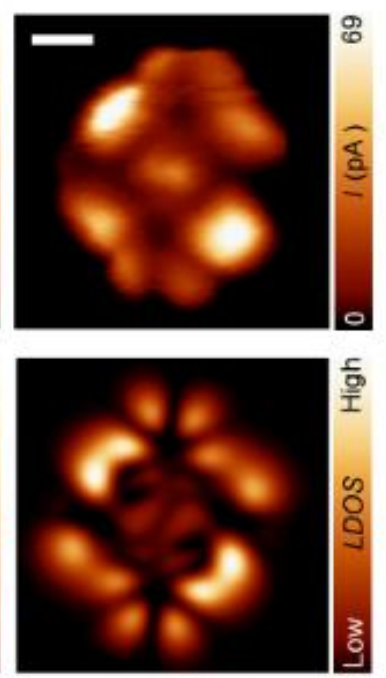

LUMO

Figure 2. Electronic structure of a close-shell porphyrin with two embedded pentagon rings. (a), Chemical structure of the product \#1. (b-c), Mean-field Hubbard model and spin-polarized density functional theory calculated energy spectrum of the porphyrin in (a). (d), dI/dV spectra taken at the two locations marked on the inset current image (scale bar: $0.25 \mathrm{~nm}$ ). Two resonances have been observed and assigned to the highest occupied molecular orbitals (HOMO) and lowest unoccupied molecular orbitals (LUMO). (e), Up: spatial resolved local density of states maps taken at the biases marked by dashed lines in (d); Down: Simulated density of states maps. Scale bars: $0.5 \mathrm{~nm}$. 
a

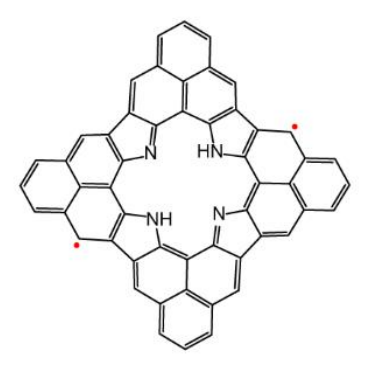

b

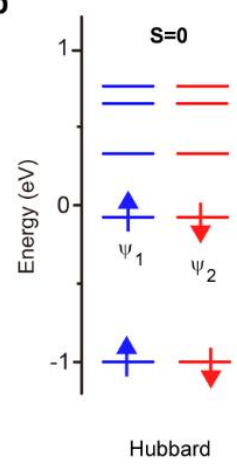

C

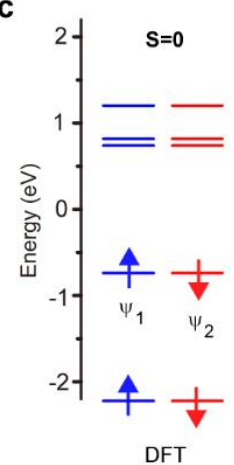

d

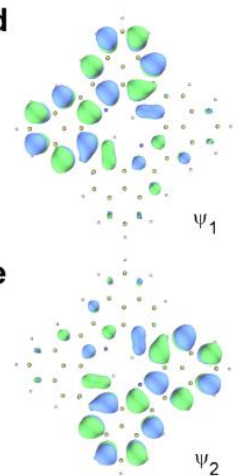

f

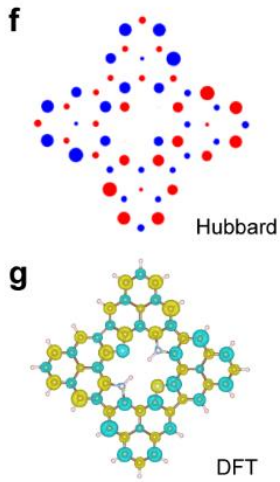

Figure 3. Spin polarized singlet ground state in the Product \#2. (a), Chemical structure of the Product \#2 with two unpaired $\pi$ electrons as marked by red dots. (b-c), Mean-field Hubbard model and spin-polarized density functional theory calculated energy spectrum of the porphyrin in (a). (d-e), Calculated frontier orbitals of the two singly occupied states as marked in (b) and (c). (f-g), Mean-field Hubbard model and spin-polarized density functional theory calculated spin density distributions. Red/green: spin up, bule/yellow: spin down. 
a

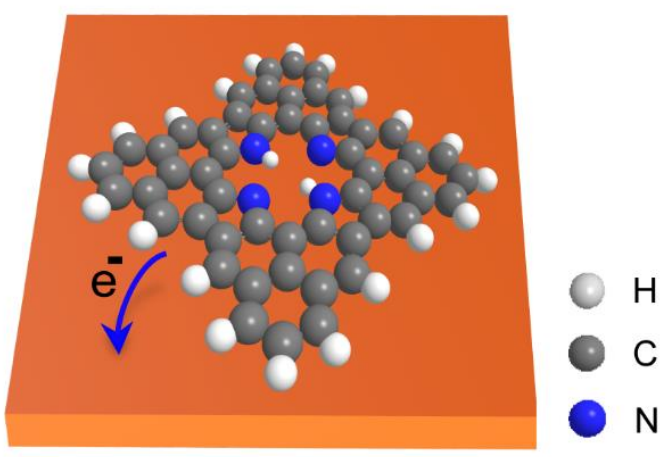

b

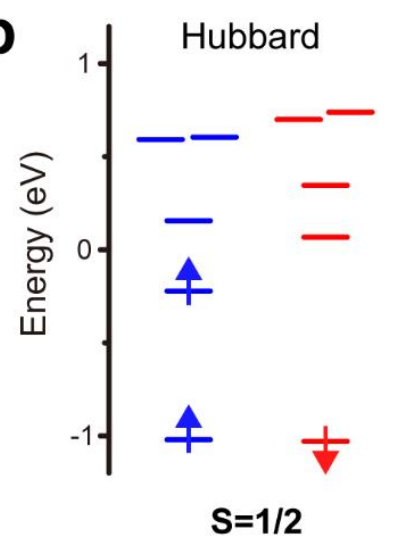

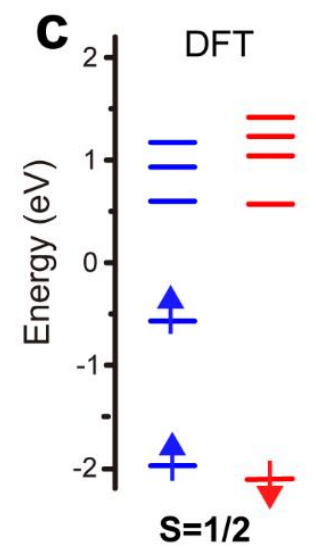

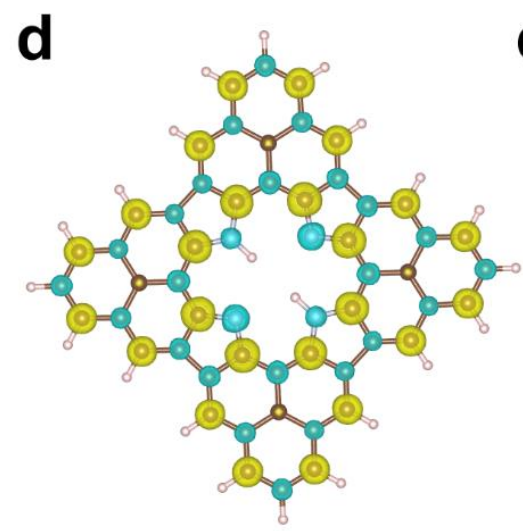

e
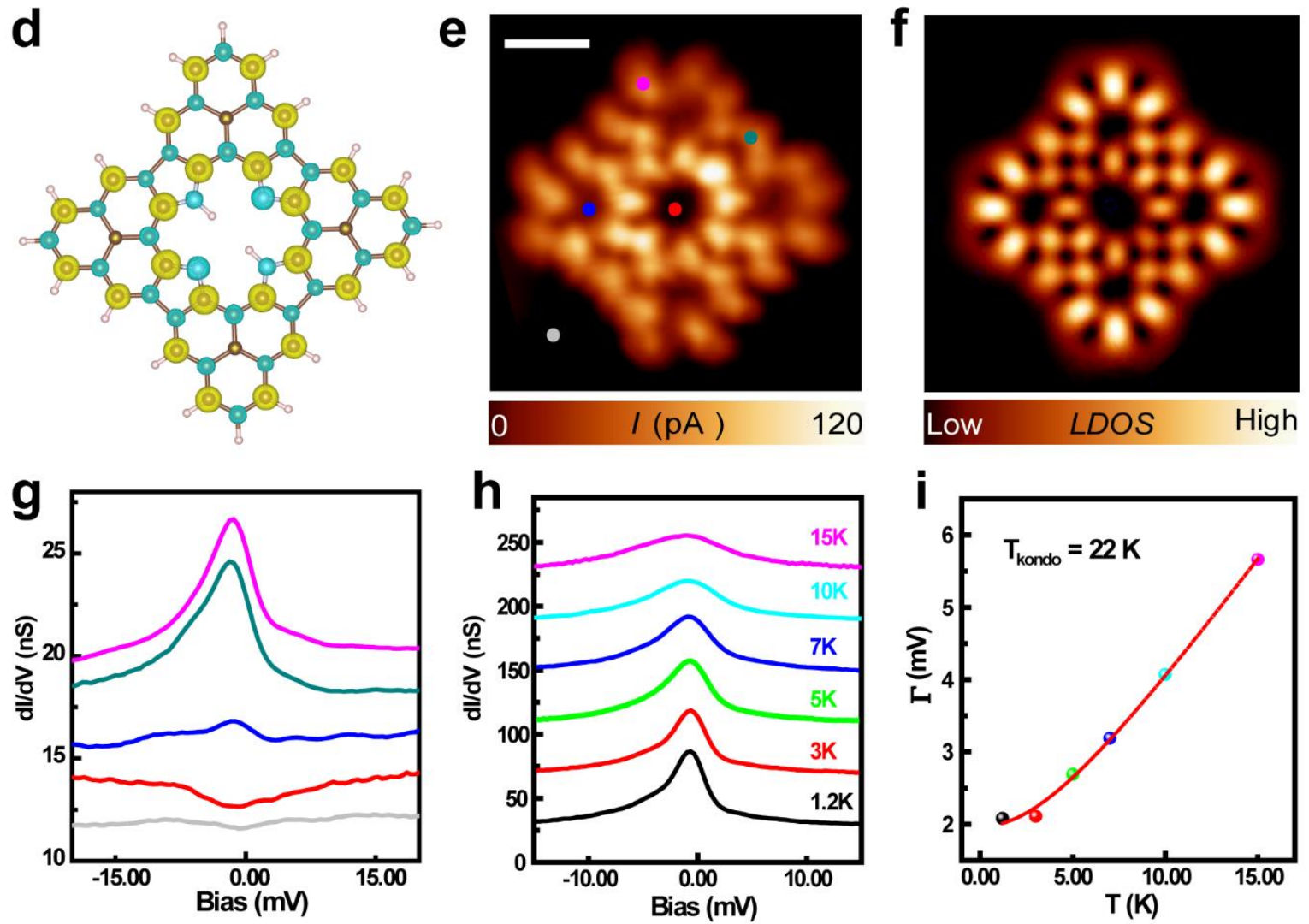

Figure 4. Electronic structure of the positively charged Product \#2. (a), Illustrative showing charge transfer from the Product \#2 to underneath $\mathrm{Au}(111)$, thus making it positively charged. (b-c), Mean-field Hubbard model and spin-polarized density functional theory calculated energy spectrum of the charged Product \#2. (d), Spin-polarized density functional theory calculated spin density distributions. Green: spin up, Yellow: spin down. (e-f), Constantheight current image (Bias voltage: $1 \mathrm{mV}$, Scale bars: $0.5 \mathrm{~nm}$ ), and simulated DOS map of the charged Product \#2. (g), $\mathrm{d} I / \mathrm{d} V$ spectra spectra taken on the locations marked in (e). (h), Temperature dependent $\mathrm{d} I / \mathrm{d} V$ spectra showing the broadening of Kondo resonace peak as increase the temperature. (i), The full-width at half maximum of Kondo resonce peak as a function of temperature. The solid red line is fitted curver giving a Kondo temperature of $22 \mathrm{~K}$. 
a

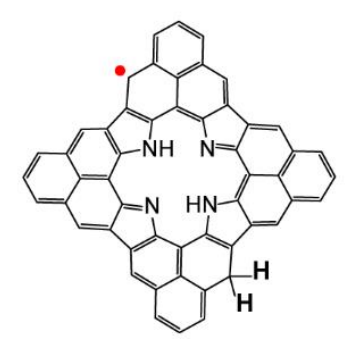

d

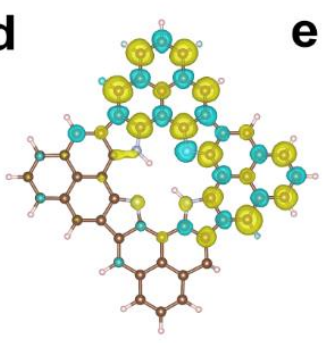

b

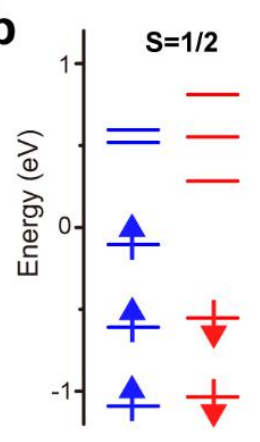

Hubbard

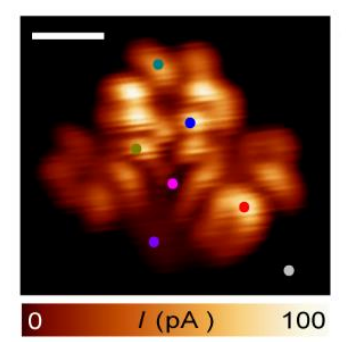

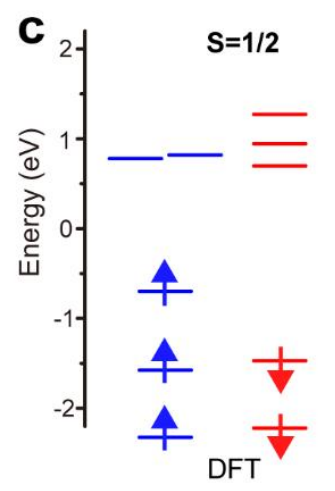

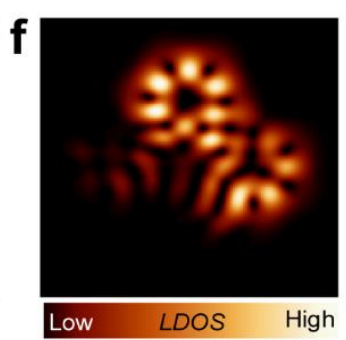

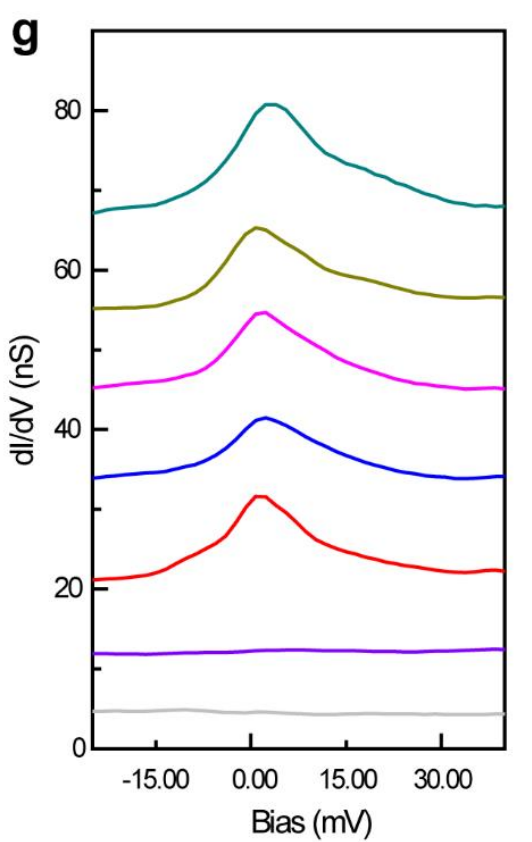

Figure 5. Electronic structure of the Product \#3. (a), Chemical structure of the Product \#3 with one unpaired $\pi$ electron as marked by a red dot. (b-c), Mean-field Hubbard model and spin-polarized density functional theory calculated energy spectrum of the Product \#3. (d), Spin-polarized density functional theory calculated spin density distributions. Green: spin up, Yellow: spin down. (e-f), Constant-height current image (Bias voltage: $1 \mathrm{mV}$, Scale bars: $0.5 \mathrm{~nm}$ ), and simulated DOS map of the charged product \#2. (g), $\mathrm{d} / / \mathrm{d} V$ spectra spectra taken at the locations marked in (e). 


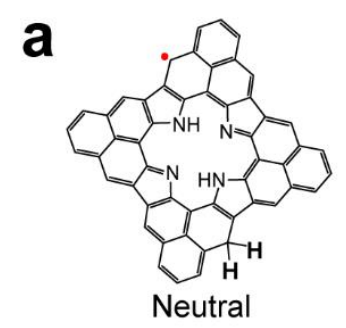

b

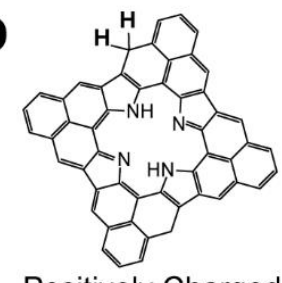

Positively Charged

C

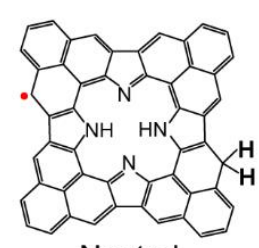

Neutral
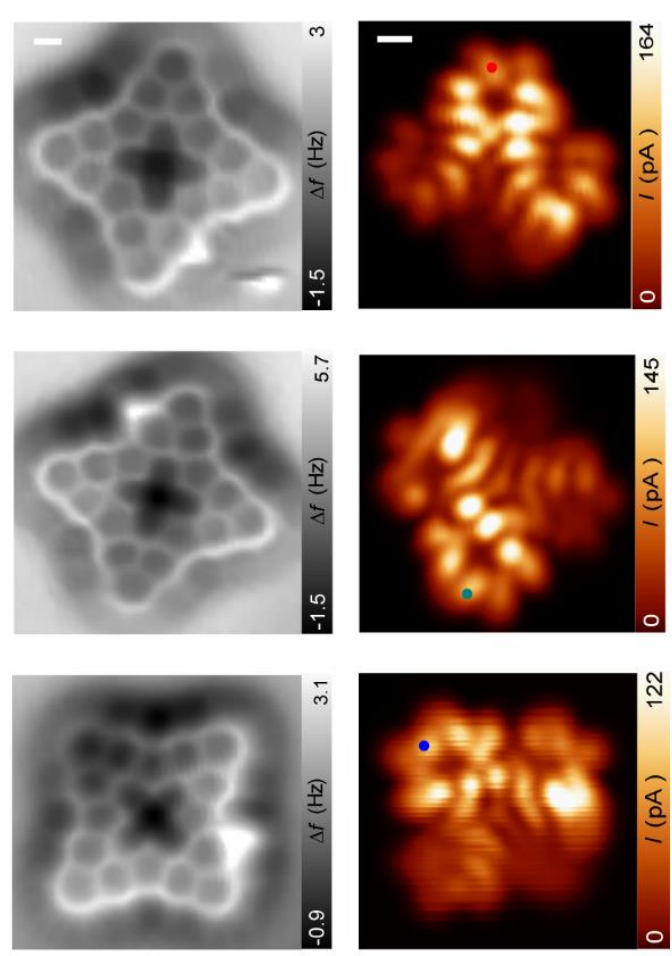

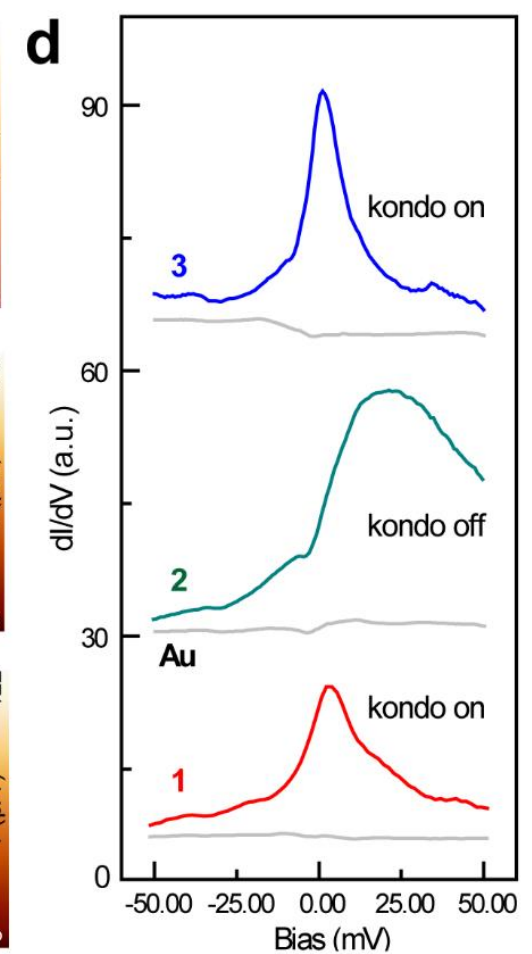

Figure 6. Switching magnetic state by STM manipulation. (a-c), From left to right: Chemical structure, nc-AFM image (Resonant frequency: $26 \mathrm{KHz}$, Oscillation amplitude: $80 \mathrm{pm}$, Scale bars: $0.2 \mathrm{~nm}$ ), Constant-height current image (Bias voltage: $1 \mathrm{mV}$, Scale bars: $0.25 \mathrm{~nm}$ ) of the Product \#3 after two manipulation steps. (d), $\mathrm{d} / / \mathrm{d} V$ spectra taken at the marked positions in (a-c). 
TOC Figure

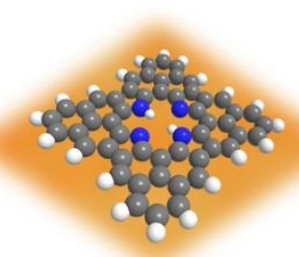

Metal-free porphyrin

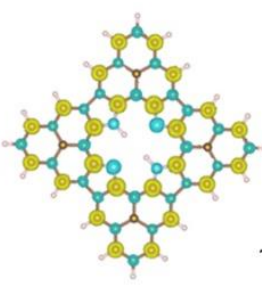

Delocalized $\pi$ radical

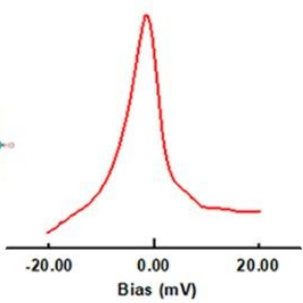

Kondo resonance 